\title{
Dissection of the Left Coronary Artery after Surgical Aortic Valve Replacement
}

\author{
Haval Sadraddin ${ }^{1} \quad$ Ulrich Krüger ${ }^{2}$ Jochen Börgermann ${ }^{1} \quad$ Mustafa Gerçek, MD ${ }^{1}$ (1) \\ ${ }^{1}$ Clinic for Cardiovascular Surgery, Herzzentrum Duisburg, Duisburg, \\ Germany \\ ${ }^{2}$ Clinic for Cardiology and Angiology, Herzzentrum Duisburg, \\ Duisburg, Nordrhein-Westfalen, Germany \\ Address for correspondence Mustafa Gercek, MD, Clinic for \\ Cardiovascular Surgery, Herzzentrum Duisburg, Gerrickstraße 21, \\ Duisburg 47137, Germany \\ (e-mail: mustafa.gercek@evkln.de).
}

Thorac Cardiovasc Surg Rep 2021;10:e52-e54.

\begin{abstract}
Keywords

- ischemia/reperfusion

- myocardial protection/ cardioplegia

- surgery

- complication

Our report presents a 73-year-old female patient with severe aortic stenosis who was admitted to our department for a surgical aortic valve replacement. After an uneventful surgery, a worsening low cardiac output syndrome with signs of myocardial ischemia occurred. Immediate angiography revealed a diffuse left coronary dissection starting from the ostium extending to the periphery of the left coronary system. The diffuse nature of the dissection ruled interventional management out and thus has been treated with urgent coronary bypass surgery. However, after an antecedent favorable course, the patient died 2 months later due to pneumonia resulting in septic shock.
\end{abstract}

\section{Introduction}

Intraoperative myocardial protection with antegrade cardioplegia by selective coronary cannulation is a widely established and safe technique used in aortic valve surgery. Injuries to the coronary arteries due to the selective cannulation are a rare but feared complication. ${ }^{1,2}$ In this case report, we present an interesting case of diffuse coronary dissection from the ostium to the periphery of the left coronary system after surgical aortic valve replacement.

\section{Case Report}

A 73-year-old female patient with severe aortic valve stenosis was admitted to an elective surgical aortic valve replacement with a Society of Thoracic Surgeons score of $1.62 \%$. Exclusion of a concomitant coronary artery disease was proven by coronary angiography ( - Fig. 1A). Transthoracic echocardiography revealed a preserved left ventricular ejection fraction and a thickened bicuspid anatomy of the aortic valve with an opening area of $0.9 \mathrm{~cm}^{2}$ and maximum and mean pressure gradients of 73 and $44 \mathrm{mmHg}$, respectively.

After induction of the general anesthesia, a median upper mini-sternotomy was performed with subsequent initiation of cardiopulmonary bypass. After aortic crossclamping, a blood cardioplegia solution was delivered to the aortic root with successful cardiac arrest. After horizontal aortotomy, antegrade cardioplegia was repeated every 20 minutes via selective coronary cannulation using coronary perfusion cannulae (SORIN GROUP, $3.0 \mathrm{~mm}$ ). After aortic valve replacement with a bioprosthesis (Epic ${ }^{\mathrm{TM}}$ Supra Valve $23 \mathrm{~mm}$, Abbott Cardiovascular, Plymouth, USA), successful weaning from the cardiopulmonary bypass was achieved under slight inotropic support without any signs of myocardial ischemia.

Four hours after surgery, the patient developed hemodynamic deterioration. Electrocardiogram (ECG) controls received

April 1, 2021

accepted

April 30, 2021
DOI https://doi.org/ 10.1055/s-0041-1731275. ISSN 2194-7635.

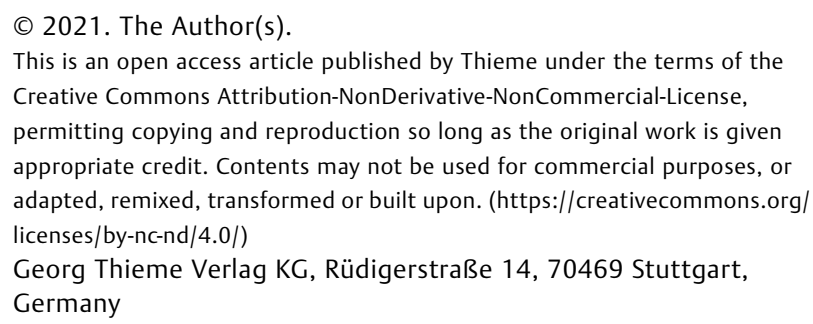



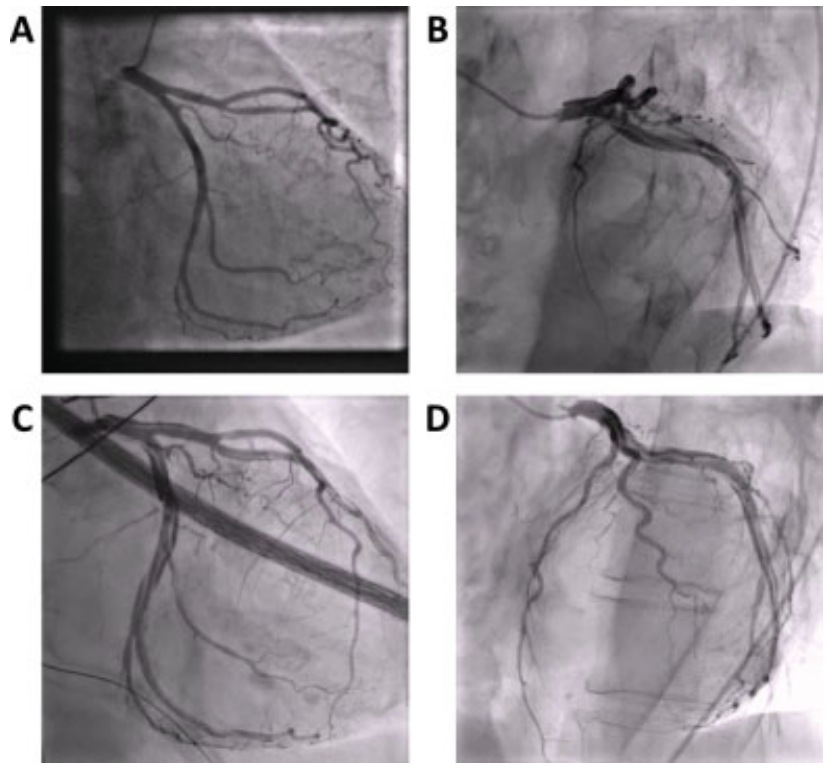

Fig. 1 (A) The preoperative angiography of the left coronary artery (LCA), revealing a nondiseased anatomy. (B-D) The postoperative angiography with dissection of LCA. Dissection begins in the left main artery with the suspected entry. All marginal branches of the circumflex artery are affected. Dissection follows the left anterior descending artery until the bifurcation of the first diagonal branch, continuing in this branch.

showed slight ST-segment elevation in V2-4 with marked elevation of cardiac biomarkers. Transesophageal echocardiography demonstrated apical and anterior akinesia that eventually led to urgent coronary angiography. The angiography revealed a diffuse left coronary artery (LCA) dissection, beginning at the left coronary ostium spreading to the distal branches (-Fig. 1B, - Videos $\mathbf{1}$ and 2). Regarding the left anterior descending (LAD) artery, the dissection continued into the first diagonal branch, sparing the distal part of the LAD ( - Fig. 1B-D). The RCA was intact. Interventional treatment by stent implantation was discarded due to the complex character of the dissection challenging identification of a suitable position in the true lumen. The decision was made to restore myocardial perfusion by coronary artery bypass grafting to the distal segment of LAD and an obtuse marginal branch of the circumflex artery.

\section{Video 1}

Coronary angiography: Left coronary artery (LCA) in anteroposterior caudal 30 degrees. Dissection beginning at the left main artery extending to both the LAD and the circumflex system. Online content including video sequences viewable at: https://www.thiemeconnect.com/products/ejournals/html/10.1055/s0041-1731275.

\section{Video 2}

Coronary angiography: Left coronary artery in right anterior oblique (RAO) 30 degrees; caudal 20 degrees. The circumflex system is affected to all marginal branches. Left anterior descending (LAD) dissection follows the first diagonal branch sparing out the distal LAD. Online content including video sequences viewable at: https://www.thieme-connect.com/products/ ejournals/html/10.1055/s-0041-1731275.

Postoperatively, a gradual hemodynamic stability with a rapid decline of cardiac biomarkers was noted, with subsequent successful termination of inotropic agents. However, after the antecedent favorable recovery, she suffered from respiratory insufficiency resulting in reintubation and tracheotomy that were followed by the development of severe pneumonia and septic shock. Unfortunately, the patient died 61 days after the operation.

\section{Discussion}

Coronary artery dissection is a rare but potentially fatal disease ensuing from spontaneous or iatrogenic origin that is often associated with coronary angioplasty and heavily affected coronary arteries with an incidence of 0.07 to $0.1 \% .^{1-3}$

Our report presents an extensive LCA dissection following a surgical aortic valve replacement. Iatrogenic coronary intimal laceration following cardiac surgery has been occasionally reported, though with no described incidence. ${ }^{3}$ In this case, selective coronary cannulation was used for cardioplegia perfusion, which, along with coronary reimplantation and resection of calcic aortic roots, is a described potential risk factor for coronary dissection. ${ }^{2,4}$

The treatment of coronary dissections depends on the resulting cardiac malperfusion and symptoms. This ranges from a conservative treatment for asymptomatic patients to a catheter-based intervention or an urgent coronary artery bypass operation. ${ }^{3}$ While the previously reported cases experienced catastrophic outcomes with early death, other recent reports have shown better outcomes that may be associated with current advances in coronary artery management. ${ }^{2,4}$ In our patient, an interventional approach was not suitable. Due to the worsening low cardiac output syndrome, urgent surgical myocardial revascularization was the only choice remaining. Fortunately, the dissection spared the distal LAD, enabling revascularization of the anterior wall segment.

The current case corroborates the liberal strategy of early postoperative coronary angiography if a hemodynamic deterioration, ECG or echocardiographic alteration, or an obvious increase in cardiac biomarkers occurs. This may help to facilitate early detection of such an iatrogenic coronary 
e54 Dissection of the LCA after Surgical Aortic Valve Replacement Sadraddin et al.

dissection and to enable in-time life-saving management and thus to minimize the often-fatal subsequent outcome. ${ }^{4}$

Disclosures

None.

\section{Funding}

No funding was received.

Conflict of Interest

None.

\section{References}

1 Hokken RB, Foley D, van Domburg R, Serruys PW. Left main coronary artery dissection during percutaneous coronary intervention treated by stenting. Neth Heart J 2002;10(10):395-398

2 Nakao K, Sawai T, Nakahira J, Hamakawa A, Ishii H, Minami T. Left main coronary artery dissection during aortic valve replacement. Anesth Analg 2017;124(06):1789-1791

3 Eshtehardi $\mathrm{P}$, Adorjan $\mathrm{P}$, Togni $\mathrm{M}$, et al. Iatrogenic left main coronary artery dissection: incidence, classification, management, and long-term follow-up. Am Heart J 2010;159(06): 1147-1153

4 Berroya RB, Mannix EP Jr. Coronary artery dissection during aortic valve operation. Ann Thorac Surg 1970;9(05):468-473 